\title{
Intelligent agent approaches for internet of things challenges: a review
}

\author{
Abstract \\ Internet of Things (IoT) is a research field that develops communications and \\ possesses many challenges although it is the development of communications. \\ Artificial Intelligence (AI) has many techniques such as Multi-agent system (MAS), \\ Artificial Neural Networks (ANNs), Fuzzy Systems (FS), Genetic Algorithms (GA) \\ these techniques should be considered in the future of IoT as they have high processing \\ computing.
}

Keywords: AI, MAS, Agent, ANNs, FS, IoT, M2M, SA, GA, RFID
Volume 4 Issue I - 2018

\author{
Hamdy AM Sayedahmed \\ Central Metallurgical Research and Development Institute, \\ Egypt \\ Correspondence: Hamdy AM Sayedahmed, Central \\ Metallurgical Research and Development Institute, Egypt, \\ Email hamdi@cmrdi.sci.eg
}

Received: November 23, 2017| Published: February 07, 2018

Abbreviations: RFID, radio frequency identification device; AI, artificial intelligence; MAS, multi-agent system; ANNs, artificial neural networks; FS, fuzzy systems; GA, genetic algorithms; BDI, belief-desire-intention; FL, fuzzy logic; SA, swarm intelligent.

\section{Introduction}

Internet of Things (IoT) is a network that uses internet services and contains surrounded different objects such as mobile nodes, smart homes, smart transport system, and many other smart systems that will lead to smart internet. Also, IoT can be defined as a machine to machine (M-to-M) connection as any node connects to another node through internet and allow other nodes to connect. The main characteristics of these objects can be addressed in having physical body, having identifier, having computing and processing capabilities, linked with least one name and address, may sense physical phenomena or trigger actions. Simply, IoT has different meanings that can be summarized as combination of "sensors and actuators" or "Sensor Networks and Near Field Communication using Radio Frequency Identification Device (RFID) tags". ${ }^{1}$ IoT is a promising approach to construct a global network that possesses smart computing objects. ${ }^{2}$ Smart computing objects will perceive from their environments and actuate with people or machines. However, the promising approach faces numerous challenges ${ }^{3}$ such as

a. Security and privacy which is responsible for confidentiality, authentication, validation, non-repudiation and integration. No all IoT Objects support powerful security methods like encryption. Therefore, security can be implemented in two levels. The first level is communication through internet. The second level is securing topology from new machines.

b. Managing data as number of smart objects increase, the amount of data increase as well. These data will be stored, processed, and exchanged among smart objects. Therefore, finding an approach to bring, search, and abstract these data should concern the amount of data size.

c. Accountability which is the governance of a global resource is not owned by single group. i.e. a statement between all IoT stakeholders is needed.

d. Trust which is a general definition of large number of different contexts. In IoT framework, the need of moving away from static classical trust management should be addressed as the dynamic of IoT platform topology

e. Different Object types, as the connection over internet will contain different objects from different vendors this will lead to develop communication protocols.

Due to writing a program code for large number of objects is such complex, it is much easier to write a code for each individual and let them control from their interactions as in smart objects. Therefore, Artificial Intelligent (AI) and Multi-agent system (MAS) are significant methods for dealing with distributed systems with different platforms which have heavy process or threats like in IoT. In MAS, Belief-Desire-Intention (BDI) model is embedded to implement artificial intelligent agent. Other, embedded fuzzy logic models (FL), artificial neural network (ANN) models, Genetic Algorithms (GA) individually or grouped.

\section{Methodology}

Most of previous researches on IoT were investigating the IoT with respect to its challenges, visions, applications and trends. ${ }^{5,6}$ Also, researches present an open question for the future of IoT spreading and the ability to simulate this global environment. In addition, it was concluded that no research can cover the full set of functionalities to meet the requirements of IoT platform. i.e. the proposed smart home, smart vehicle, smart city...etc. applications cannot be fully covered in a simulated environment. Although the IoT framework varies from physical geographic position, device types, and used protocols for data exchange. AI and MAS can introduce a solution for such situation when embedding agents to smart objects and let control to be through agents.

\section{Artificial intelligent solution}

MAS are a system of agents group. Agents are autonomous objects which perceive and actuate from their environment to achieve certain goals. $^{6-8}$ The agent is placed in an environment and able to perceive information about it. Moreover, agent can be either a software or hardware. Thus, it may be exploited that it is software and be the communication protocol. Therefore, the method to interpret the perceived information should be considered. Artificial Intelligent as a research field includes many sub-fields such as 
Artificial Neural Networks (ANN), Fuzzy Logic (FL), Machine Learning, Genetic Algorithm (GA), and Robotics ... and so on. ANN, FL, and GA are presented since they have high processing computing. ANN is one method for interpreting the information, it was been inspired by the biological neural networks. ANNs provide a faster method of developing systems that classify threats events such as in character recognition, traveling salesman's problem...etc. $\mathrm{FL}$ is another method for translating the perceived information. Also, fuzziness can be found in complex systems that hard to be modeled as in IoT. FL uses the concept of partial truth of facts. FL was used in facial pattern recognition, washing machines, vacuum cleaners and many other applications. GA is another approach in AI which provides high quality solution to optimization and search problems. Genetic programming, automated trading systems, airlines revenue management are applications based on GA.

\section{Discussion}

AI has many fields that fit in complex systems models such as ANNs, FL, and Swarm Intelligent (SA). IoT as a new environment has a challenges such as security, privacy, data exchange, accountability, and trust. These challenges will be tested and simulated by researchers thus; the need to construct an aware-simulator should be addressed. It may be a simulator that partially simulates the IoT environment but no fully simulator to it. IoT can be treated as a network of agents or a network of robots and hence the ability to enable AI techniques will be considered. Therefore, growing human agents will arise.

\section{Conclusion}

In this quick review, the IoT challenges was reviewed and discussed. Also, the presented question after reviewing is the ability of IoT environment to be simulated and thus the ability of its expansion. AI various techniques introduce a solutions for complex system models and it have well known applications such as smart home and smart vehicle. The vision of the future of IoT will consider MAS as a mean to its solutions. In addition the AI different techniques.

\section{Acknowledgments}

None.

\section{Conflicts of interest}

None.

\section{References}

1. Andrew Whitmore, Anurag Agarwal, Li Da Xu. The Internet of Things-A survey of topics and trends. InfSyst Front. 2015;17(2):261-274.

2. Soma Bandyopadhyay, Munmun Sengupta, Souvik Maiti, et al. Role of Middleware for Internet of Things: A Study. International Journal of Computer Science \& Engineering Survey. 2011;2(3):1-12.

3. Andreas Schmidt Jensen. Multi-Agent Systems: An Investigation of the Advantages of Making Organizations Explicit. In: Kongens Lyngby, editor. 2010. p. 1-136.

4. Xu Li, Rongxing Lu, Xiaohui Liang, et al. Smart Community: An Internet of Things Application. IEEE Communication Magazine. 2011.

5. Daniele Miorandi, Sabrina Sicari, Francesco De Pellegrini, et al. Internet of Things: Vision, applications and research challenges. Ad Hoc Networks. 2012;10(7):1497-1516.

6. Han Yu, Zhiqi Shen, Cyril Leung. From Internet of Things to Internet of Agents. International Conference on Green Computing and Communication and IEEE Internet of Things and IEEE Cyber, Physical and Social Computing. 2013.

7. Taboun MS, Brennan RW. An Embedded Multi-Agent Systems Based Industrial Wireless Sensor Network. Sensor (Basel). 2017;17(9):E2112.

8. Andries P Engolbrecht. Computational Intelligence. An Introduction. 2nd edn. John Wiley \& Sons. 2007. 